\title{
Treatment Outcome and Associated Factors among Type 1 Diabetic Children<15 Years Admitted With DKA in Bahir Dar City Public Referral Hospital, North West, Ethiopia: A retrospective Cross Sectional Study
}

Fentahun Meseret ( $\square$ mesie1055@gmail.com )

Haramaya University

\section{Research Article}

Keywords: type 1 diabetes mellitus, diabetic keto acidosis, treatment outcome, children, Ethiopia

Posted Date: November 10th, 2021

DOI: https://doi.org/10.21203/rs.3.rs-981711/v1

License: @ (i) This work is licensed under a Creative Commons Attribution 4.0 International License. Read Full License 


\section{Abstract}

Background: Length of hospital stay and overall in hospital mortality of diabetic keto acidosis during management are the foremost primary out comes that should be measured. Because, it is highly trusted on the improvement of diabetic keto acidosis related complication management. However, data associated to length of stay, mortality rate due to diabetic keto acidosis remains to be limited in Ethiopia.In addition to this, Nonfiction is much scarce in relation to factors associated with treatment outcome starting from its initial presentation and the overall management process.

Objective: To assess treatment outcome of children< 15 years old admitted with diabetic keto acidosis at Felege Hiwot comprehensive referral hospital, North West, Ethiopia, 2021

Methods: Retrospective cross sectional study was conducted at Felege Hiwot comprehensive referral hospital among randomly selected sample of 176 children admitted with diabetic keto acidosis from 2016 to 2021.Data were collected by using data abstraction tool. Then it was coded, enter, cleaned and stored into Epi-data version 4.2 and was exported into STATA 14.0 statistical software for analysis. Categorical variables were described using proportions and compared using Chi-square test; whereas continuous parametric variables with mean and standard deviation and compared using parametric (t-test). Model goodness-of-fit and assumptions were checked. Finally, association between independent variables and length of hospital stay were assessed using binary logistics regression and Variables with $p$-value $<0.05$ were considered as statistically significant.

Result: The mean length of hospital stay was $9.5 \pm 6.2$ days. About $59.3 \%$ of had long hospital stay (>7days).Majority of the clients (97.5\%) were improve and discharged with $14.2 \%$ management complication and $4(2.5 \%)$ died in the hospital. Factors that affect long hospital stay were residence(AOR=4.31;95Cl=1.2514.80 ), family history of diabetes ( $\mathrm{AOR}=0.12 ; 95 \% \mathrm{Cl}=0.02-0.64)$, glycaemia at admission ( $\mathrm{AOR}=1.01 ; 95 \% \mathrm{Cl}=1.00-1.02)$,insulin skipping (AOR=0.08;95\% $\mathrm{Cl}=0.01-0.98)$,abdominal pain $(A O R=4.28 ; 95 \% \mathrm{Cl}=1.11-15.52)$, time in which the patient get out of diabetic keto acidosis(AOR=6.39;95\% Cl=1.09-37.50).

Conclusion and Recommendation: Majority of clients were showed improvement and discharged to their home after longer hospital stay and with very low mortality rate followed by complication (14.2\%). The time with in which the majority of the clients get out of DKA were found in between $24-48$ hours. Thus, to achieve the intended treatment out come early in time, modification of the approach by emphasizing the above predictors is mandatory with the recommendation of multicenter study in this regard.

\section{Introduction}

Type 1 diabetes militias (T1DM) is the most common endocrine metabolic disorder among children(1).Almost one in 300 children develop T1DM(2).And these finding have been documented in many countries including both developed and developing countries(2),(3). It has been reported that the incidence of type 1 diabetes is increasing by $3-4 \%$ per year globally in light of geographical variation with high correlation of this incidences to socio economic factors(4),(5).

Diabetic keto acidosis (DKA) is a serious, life threatening and expensive acute complication of T1DM that can occur when there is a comparative or complete decrease in circulating insulin levels in relation to an increase in counter governing hormone levels(6).In response to this disparity, normal physiologic mechanisms are exaggerated, resulting in hyperglycemia, hyper osmolality, ketosis, and acidosis(6),(7). It can occur under two occasions (i) at the time of T1DM diagnosis (disease first appearance) or(ii) in clients with a previous T1DM diagnosis who do not receive proper interventional follow up or who are suffering from under recurrent infection that has not been sufficiently controlled(7). The magnitude of DKA cases among diabetic children has steadily increased for the past 3 decades (8).DKA and its complications are the most common cause of hospitalization, morbidity and mortality in children with established diagnosis of T1DM(9).

Insulin deficiency, dehydration and hormone increment such as cortisol, adrenaline, glucagon and growth hormone are some of the clinical feature of children admitted with DKA(10). The biochemical criteria for the diagnosis of DKA are hyperglycemia(blood glucose level $>200 \mathrm{mg} / \mathrm{dl}[>11.1 \mathrm{mmol} / \mathrm{L}]$ ), venous $\mathrm{pH}$ lessthan7.3 or serum bicarbonate level less than $15 \mathrm{mEq} / \mathrm{L}(<15 \mathrm{mmol} / \mathrm{L}$ and ketonemia (blood b-hydroxybutyrate concentration++3 mmol/L ) or moderate or severe ketonuria(6),(11),(12).

Patients can dramatically ill, the severity of presentation can be graded based on the degree of acidosis ;mild DKA: venous pH from 7.2-7.3or serum bicarbonate $<15 \mathrm{mmol} / \mathrm{L}$; moderate DKA: venous $\mathrm{pH}$ from 7.1-7.2 or serum bicarbonate $<10 \mathrm{mmol} / \mathrm{L}$; sever DKA: venous $\mathrm{pH}<7.1$ or serum bicarbonate $<5$ $\mathrm{mmol} / \mathrm{L}$ and as severity increases, the need for pediatric intensive care as well as risk of morbidity and mortality increases(13).But, with aggressive management most patients can recover rapidly(8).

In spite of numerous advances in the management of type one diabetes mellitus, more than 70\% of T1DM patients unable to maintain glycemic control(14).As a result, diabetic keto acidosis(DKA)became the most common sticky situation of T1DM in children with an incidence of 1-10\% of diabetic clients per year in developed nations(7),(20).In countries such as Germany, Europe, north America and Australia the average rate of DKA ranges from 30 to $70 \%$ in the child diagnosed with T1DM(15), whereas, the proportion of DKA in Asian children among the total cohort was 33.6\%(16).

The prevalence of DKA at the time of diagnosis in sub-Saharan Africa was between 70 to $80 \%$.(17).The inclusive proportion of children diagnosed with DKA in Addis Ababa Ethiopia was high(35.8\%)(18).Likewise in Gojjam Ethiopia the overall incidence rate of DKA was 2.27 per 100 children/month of observation(19).

DKA is the most common cause of acute comorbidity and mortality in children with T1DM with the range of 16.5 to $78 \%$ admission rate in hospital(15). With the average length of recovery time between 24 hours- 8 days $(8,10)$.where a low pH and high total leucocyte count (TLC) at admission were observed to be associated with prolonged acidosis (21).The pooled mortality rate for DKA is estimated to range from $2-5 \%$ in developed nations and $6-24 \%$ in developing countries(22),(23). 
It has been identified that, poor glycemic control, comorbidities, younger age and low socio economic status have been associated with increased risk of developing DKA , but it is unclear if this risk factors influences patient outcomes while in the pediatric management center(24).

Higher mortality rates among children admitted with DKA were reported with an increased incidence of cerebral edema, sepsis, shock, cerebral injury, altered level of consciousness ,respiratory failure with hyposphotemia, renal failure and delayed diagnosis both in developed and developing countries,(23),(25),(29), (30).DKA can be principal source of death, mainly when complicated by cerebral edema/injury with an estimated mortality rate of 20 to $50 \%$ and $15-35 \%$ of survivors are left with permanent neurologic deficit(20),(21),(31).Acute complication of DKA may be accompanied by malnutrition, parasitic and microbial infections with tuberculosis and HIV (31). Over lapping of these condition contribute to the increased rate of morbidity and mortality. In ability of the patients to afford insulin treatment leads to poor glycemic control as a result , patients may seek alternative treatment from traditional healers or use herbal remedies further complicating the management process(32).

The DKA management protocol which can be used in pediatrics clinical care unit was prepared by the pediatric endocrine and diabetes associations in 2016 based on the international society for pediatric and adolescent diabetic (ISPAD) and European society for pediatric endocrinology (ESPE) guidelines (12),(15). DKA management is too challenging as it can be associated with different types complication. Accessing and utilizing endocrine and critical care published guidelines and attempt to set standards in DKA diagnosis and management is required by taking in to consideration that, standard treatment guidelines for DKA at present may not be ideal as they are adapted from those of the developed world(32).

During DKA treatment, administration of insulin inhibits the production of ketoacidosis and facilitates their metabolism, thereby helping in correcting the acidosis(10).Effective treatment requires the replacement of insulin ,fluid and electrolytes in addition to applying the novel approach of early diagnosis and treatment by multidisciplinary diabetic care team to ensure good outcomes(28),(33).Length of hospital stay (LHS) and overall in hospital mortality (IHM) among DKA children during management are the foremost primary out comes that should be measured. Because it is highly trusted on the improvement of T1DM and DKA related complication management. However, data associated to LHS the rate of IHM due to DKA remains to be limited in Ethiopia. In addition to this, Nonfiction is much scarce from Ethiopia in relation to factors associated with treatment outcome among children admitted with DKA starting from its initial presentation and the overall management process.

Therefore, the current study is aimed to determine treatment outcome of children< 15 years old admitted with DKA at Felege Hiwot comprehensive referral hospital in Amhara region, North West, Ethiopia

\section{Methods}

\section{Study area and period}

The study was conducted in Bahir Dar city; located $565 \mathrm{Km}$ far from Addis Ababa, the capital city of Ethiopia, at Amhara national regional state, North West Ethiopia. In Bahir Dar city, there are two public referral hospitals, one primary hospitals, ten health center and four private hospitals. And this study was conducted in Felege Hiwot comprehensive specialized referral hospital (FHCSH). This hospital can be expected to serve for more than 10 million populations coming from Bahir Dar city, west Gojjam zone, east Gojam zone, awi zone, north and south wollo zones, south\& north Gondar zones, partial part of Benshangul Gumuz and Oromia region. FHCSH has currently a total of 1431 man power in each discipline with 500 formal beds, 11 wards, 39 clinical and non-clinical departments /service unit / providing Diagnostic, curative, Rehabilitation and preventive service at outpatient \&inpatient based.

Apart from other services, this referral hospital provide diabetic treatment services by nurse practitioners, pediatrics residents and pediatricians.

The study period address from1stJanuary, 2016 to February 30 /2021.

\section{Study design}

An institution based retrospective cross sectional study was employed.

\section{Source Population}

All pediatrics T1DM patients who were on the follow up at Felege Hiwot comprehensive referral hospital were source of population in this study.

\section{Study Population}

The study population were all pediatrics T1DM patients who were admitted due to DKA in the past before the study period.

\section{Sample Size and Sampling Technique}

Randomly selected Pediatric cases ( $\leq 15$ years old) T1DM patients admitted with DKA in the period from Junuary ${ }^{\text {st }} 2016$ to February 30,2021 (5-years) were included. To determine the sample size, the following assumptions were considered: $80.95 \%$ of clients were discharged with improvement in Jimma with $95 \%$ confidence level and 5\% marginal error(22). The sample size was calculated by using Raosoft software (http://www.raosoft.com/samplesize.html). Since the total population is less than 10,000, i have used correction formula. Based on this, the final sample size was became 176.

Page $3 / 19$ 


\section{Sampling Procedure}

The study participants were selected from the registration book. The medical records of children who were admitted with DKA from January $1^{\text {st }} 2016$ to February30, 2021 were selected. Finally, cards that fulfilled the criteria were reviewed.

\section{Inclusion Criteria}

Children age less than 15 years old and diagnosed with DKA from January 1/2016 to February 30/2021 was included

\section{Exclusion Criteria}

Children's chart which had incomplete information and lost during the study period were excluded from the study.

\section{Dependent Variables}

Length of hospital stay and overall in hospital mortality of DKA

\section{Independent Variable}

Socio demographic (age, gender, residence, educational status of the children including weight and height ...) and institution related variables (diabetic care team,....); Diabetic related variables/clinical and biochemical variables (family history of DM, type of DM, severity of DKA, admission blood glucose readings(RBS mg/dl), duration of chief compliant, symptoms at presentation, comorbidities, number of comorbidity, types of comorbidity, all vital sign, DKA precipitating factors, frequency of DKA episode, electrolyte disturbance, ketone and urine glucose level and Treatment related variables and/ or medication related variables(co medication and therapeutic class of co medication.)

\section{Operational Definitions}

Diabetic keto acidosis(DKA): Is defined as admission blood glucose $>250 \mathrm{mg} / \mathrm{dl}$ and presence of ketonemia and/or ketonuria (6),(11),(12)

Hyperglycemia: Is defined as random plasma glucose level>200mg/dl and hypoglycemia is defined as a blood glucose level less than or equal to 70 $\mathrm{mg} / \mathrm{dl}$. (13)

Euglycemia: Is defined as serum glucose of between 100 and $200 \mathrm{mg} / \mathrm{dl} .(51)$

Normoglycemia: Is defined as blood glucose level of between $90 \mathrm{mg} / \mathrm{dl}$ and $130 \mathrm{mg} / \mathrm{dl}(12)$

Long hospital stay: Was defined as hospital stay for more than seven days and short hospital stay was considered if the patient stay in the hospital for less than or equal to 7 days.(52)

Poor glycemic control: Is defined as a serum glucose rebound at least one times while the patients are on DKA treatment.

Treatment outcome: The length of hospital stay and in hospital mortality of DKA were the measure of treatment out come in the context of present study.

\section{Definition Terms}

Chief complaint: Is main reason that lead the person to visit hospital.

Co-morbidity: Implies concomitant diseases which are not the complications of DM and/or DKA.

Co-medications: Are concurrent medications prescribed for treatment of diseases, deficiencies, other than antihyperglycemics.

Diabetic ketoacidosis: Implies patients with positive urine and/or serum ketone and with plasma glucose greater than $250 \mathrm{mg} / \mathrm{dl}$.

DKA severity(38)

Mild DKA: Urine and/or serum ketone positive with plasma glucose greater than 250mg/dl, arterial pH 7.25-7.30, and with an alert level of consciousness.

Moderate DKA: Urine and/or serum ketone positive with plasma glucose greater than $250 \mathrm{mg} / \mathrm{dl}$, arterial pH $7.0-7.24$ with an alert or drowsy level of consciousness.

Severe DKA: Urine and/or serum ketone positive with plasma glucose greater than $250 \mathrm{mg} / \mathrm{dl}$, venous $\mathrm{pH}$ less than 7 with stupor or coma.

Insulin defaulter: Are known T1DM patients who discontinued insulin treatment because of different reasons. 
Patient characteristics: Implies the different clinical characteristics and biochemical findings of the patient

\section{Data Collection Methods}

Medical records of patients with DKA admitted to the hospital were draw from patient logbook and drawn from card room. Selection of the medical records for sampling was based on the physicians confirmed diagnosis on patient logbooks. Participants included in the study were all diabetic patients with DKA admitted to Felege Hiwot comprehensive referral hospital with age $<15$ years old and whose medical record contained complete patient data.

\section{Data Quality Control}

The data were collected by trained data collectors using structured and pre tested data extraction tool. Data were collected on patient demographics, presenting symptoms, precipitating causes of DKA, vital signs, biochemical profiles (admission blood glucose, admission urine ketone, urine glucose) at presentation to the inpatient department, time from presentation to resolution of urine ketone, length of hospitalization and treatment outcomes.

\section{Data Processing and Statistical Analysis}

Data were entered into Epidata version 4.2 software for cleaning, and exported and analyzed using STATA 14.0.

Tables and text were used to present the findings. Categorical variables were described using proportions, continuous parametric variables with mean and standard deviation. Categorical variables were compared using Chi-square test and fisher's exact test, whereas, continuous variables were compared using parametric (t-test).

Binary logistics regression was done to determine the factors that affect length of hospital stay and mortality.

\section{Ethical Considerations}

Ethical clearance was obtained from the institutional review board of Bahir Dar University (IRB number 01-008). Written supportive letter was taken from pediatrics department of the hospital on behalf of the patients. This study has no any danger or negative consequences for the study participants. Medical record number was used for the data collection and personal identifiers of the client was not use in this research report. Access to collected information was limited to the principal investigator and confidentiality had preserved throughout the time.

\section{Results}

\section{Sociodemographic characteristics}

One hundred seventy six (176) medical records were reviewed; off which, fourteen (7.95\%) client's chart were excluded from the study due to pertinent data being missing. As a result, 162 clients were included in the study which is $92.05 \%$ in response rate.

Mean age of the study participant was $8.1 \pm 4.7$ years with 2.4 years mean duration of diabetes. And almost $48.8 \%$ of them were in the age group of $11-14$ years.

More than half of the patients were male (51.2\%) and majority of the patients (66.7\%) were from rural area. (Table 1).

\section{Clinical characteristics}

Out of the investigated clients 129(80.6\%) were newly diagnosed cases and the remaining 31(19.4\%) were known diabetic clients. Majority (103[63.6\%]) of the clients were on mixed insulin (regular and lent) treatment and the other clients were on either regular and NPH (30[18.6\%]) or NPH alone (29[17.9\%]) with an average insulin dose of $16.5 \pm 10.7 \mathrm{u} / \mathrm{kg} / \mathrm{d}$ during initiation of treatment. More than half of the patients had DM for more than three years (86[53.1\%]).And many of the clients had history of comorbid illness (108[66.7\%]).Table 2.

\section{Frequency of DKA episode, severity and its precipitating factors}

The frequency of DKA recurrence was assessed and showed that, most of the clients DKA once (116[71.6\%]), 31[19.1\%] had two episodes, 12[7.4\%] and $3[1.9 \%]$ of the clients had three and four episodes of DKA recurrence during their follow up period respectively. And 29(17.9\%) of the clients were on sever DKA whereas, $61(37.7 \%)$ and $72(44.4 \%)$ of the clients had moderate and mild DKA during their presentation. The most common precipitating factor was found to be infection (91[56.2\%]). Other precipitating factors are shown in Table 3.

\section{Clinical presentation and Laboratory results of clients admitted with DKA}


In this study most of the patients were presented with polyuria (144[88.9\%]) and polydipsia (137[84.6\%]).In addition to these, nausea/vomiting (63[38.9\%]), polyphagia (61[37.7\%]) and abdominal pain (51[31.5\%]) were reported. Regarding vital sign, the mean value of pulse rate $115 \pm 22.1$ beats/min, respiration rate $31.1 \pm 10.3 \mathrm{breath} / \mathrm{min}$ and body temperature $36.6 \pm 0.9^{\circ} \mathrm{c}$. Table 4 .shows the detail in regard to clinical presentation and some of laboratory results.

\section{Management protocol and its treatment outcome}

In this study most commonly used type of fluid bolus was found to be $0.9 \%$ normal saline (161[99.4\%]) with an average amount of $362.2 \pm 348.4$ mililiters. Maintanance fluid was carried out mostly with normal saline $(0.9 \% \mathrm{NS})$ and D5W $(75[46.3 \%])$. The average rate of fluids, in the majority of the patients were $89 \pm 14 \mathrm{ml} /$ hour. Regular insulin was the only type of insulin which is administered for all DKA clients with an average rate of $9.6 \pm 4.3 \mathrm{u}$ in every 2-4 hours and then every 6 hours, till free of ketone(standing dose). However only 56(34.6\%) of the clients were repleted with potassium chloride. And majority of clients were got out of DKA with in the first $24-48$ hours (58[35.8\%]).More than three quarters of the patient (139[85.8\%]) did not develop any type of complication; However, hypokalemia $(11[6.8 \%])$ and hyponatremia $(5[3.1 \%])$ were found to be the most common complication followed by neurologic sequel and hypernatremia both accounting four cases (4[2.5\%]), 4[2.5\%]).

The average length of hospital stay was $9.5 \pm 6.2$ days; where, majority of the clients (96[59.3\%]) were stayed in the hospital more than the expected durations (> 7 days). Concerning the overall treatment outcome, only (4[2.5\%]) clients died; whereas the other patients (158[97.5\%]) showed improvement and discharged to their home. Table 5.

Over all treatment outcome among type 1 diabetic children admitted with DKA, Bahir Dar, 2021(n=162.Figure 2

\section{Factors that determine long hospital stay among type 1 diabetic children admitted with DKA}

The independent variables such as age, residence, client educational status, BMI, duration of chief complaint, family history of DM, frequency of DKA episode, severity of DKA, DKA precipitating factor, clinical presentation of DKA like polydipsia, abdominal pain, cough and confusion, location of admission, glycaemia at admission, body temperature, having history of comorbidity such as UTI,URTI, fungal infection, Comedication, time in which the patient get out of DKA and management complication were significantly associated with long hospital stay at the point less than 0.25 level of significance from bivariable analysis.

However, only residence, family history of DM, glycaemia at admission, DKA precipitating factors ,DKA presentation such as abdominal pain, location of admission and time in which the patient get out of DKA were found to be significantly associated with long hospital stay in the multivariable logistic regression model less than $5 \%$ level of significance.

The presence of interaction among independent variables were checked by multicollinearity test but there was no significant interaction as it was confirmed by the value of variance inflation factor (VIF) which is less than ten.( Annex IV).

Consequently, after adjusting other covariates, the odds of long hospital stay among the rural resident clients were greater by $31 \%$ as compared with the urban resident groups of the client $(\mathrm{AOR}=4.31,95 \% \mathrm{Cl}=1.25-14.80$, $\mathrm{p}$-value=0.020).

Likewise, DKA presentation with abdominal pain among clients were associated with longer hospital stay by $28 \%$ as compared to clients with no symptoms of abdominal pain $(A O R=4.28,95 \% \mathrm{Cl}=1.11-15.52$, $\mathrm{p}$-value=0.035).this means, the time needed to reach on recovery and discharge to their home among clients with abdominal pain was significantly longer compared with clients with no abdominal pain during their presentation.

Furthermore, those clients get out of DKA after 72 hours had more likely to be stayed in the hospital by 6.39 times as compared with clients get out of DKA less than 24 hours $(\mathrm{AOR}=6.39,95 \% \mathrm{Cl}=1.09-37.50$, $\mathrm{p}$-value $=0.040)$

However, clients with DKA precipitated by omission of insulin had less likely to be stayed in the hospital by $92 \%$ as compared with DKA clients precipitated by infection (AOR=0.08, 95\% Cl=0.01-0.98, p-value=0.048) (Table 6)

\section{Discussion}

The purpose of this study is to determine treatment outcome of children< 15 years old admitted with DKA in Bahir Dar city Felege Hiwot comprehensive referral hospital.

In this study, majority (97.5\%) of admitted DKA clients were showed improvement and discharged to their home with 9.5 days mean length of hospital stay. This finding is much higher than the study conducted at south Asia and Jimma university hospital in Ethiopia, where only $80.5 \%, 84 \%$ showed improvement and discharged to their home respectively(22), (36).

In this study, the magnitude of mortality rate is $2.5 \%$ which is relatively higher than previously reported mortality rate from developed nations ( $0.15 \%-0.31 \%)$ (23) and mortality rate testified in Sudan which was $1.7 \%(39)$ the reason might be due to high prevalence of infection(56.2\%) and comorbidities(66.7\%) as well as treatment complications $(14,2 \%)$ in this study. But lower than the study reported in India $(8.75-12.8 \%)(21,26)$ and in other developing countries like Kenya and including Tigray region in Ethiopia $(3.4-13.4 \%)(18,23,40)$. 
Concerning hospital stay, although no clear cut off point is set about when to say there is long hospital stay for clients admitted with DKA, most of clinical experts have common consciences for longer hospital stay, if and only if, the patient stayed/admitted for more than seven days in the hospital with successful treatment by prompt correction of hyperglycemia, diabetic acidosis and electrolyte disturbances(52).Therefore, the finding of this study point out longer average hospital stay(9.5)days to be discharged from the hospital as compared with the study conducted in united states (2.5) days (35),Al-Azhar University in Gaza(5.88 \pm 2.55 ) days (38), in Kenya (8 median )days(25) and 3.7 to 3.4 days in other study; even some patients can be discharged within 24 hours of hospital admission despite the case is with sever $\mathrm{DKA}(8,44,50)$.

This discrepancy of treatment outcome basically in patient recovery, mortality rate and length of hospital stay can be due to differences in treatment protocol implemented by each health care institutions and population characteristics, sample size, study methodology and overall health care system including resource allocation.

In regard to predictors, residence of the participant was found to be significantly associated variables that determine DKA hospital stay. The study showed that, clients from rural area had 4.31 times more likely to stayed in the hospital for longer time as compared with those clients living in urban

area $(A O R=4.31,95 \% \mathrm{Cl}=1.25-14.80)$. This can be due to shortage of emergency service transportation so as to access immediate medical management(22,26), which again complicates the case(26). And the finding is supported by the study conducted in turkey (45).

Those clients having family history of diabetes mellitus had $88 \%$ less likely to be stayed in the hospital for longer time as compared with clients with no family history of diabetes(AOR=0.12,95\% $\mathrm{Cl}=0.02-0.64)$. And the reason behind is not inclusive yet.

Amount of serum glucose at admission also significantly associated with longer hospital stay. Length of hospital stay increases by one day, as serum glucose value increases by one unit $(A O R=1.01,95 \% \mathrm{Cl}=1.00-1.02)$. This could be due to, sever hyperglycemia leads to sever acidosis/dehydration, electrolyte disturbances and later altered sensorium at presentation $(26,44,45,50)$. Which again overburdened the situation by increasing the number of clients to undergo further complication as it was supported by the finding in this study.

In this study, precipitating factors of DKA by insulin skipping/omission was less likely associated with longer hospital stay by $92 \%$ as compared with those client's DKA precipitated by infection(AOR=0.08,95\% Cl=0.01-0.98).

This is because having concurrent infection has an influence on diabetes disease progress with impairment of glucose metabolism possibly lead to deterioration of glycemic control. Infection might also cause high level of counteracting hormones which triggering an episode of hyperglycemia and which may complicate fluid and electrolyte replacement therapy protocol with concurrent treatment for infectious conditions to avoid possible life-threatening drug interactions(32). The finding is supported with the studies conducted in Addis Ababa, Jimma and Kenya(18,22,25)

A client with abdominal pain during presentation is 4.28 times more likely to be stayed in the hospital than clients with no symptoms of abdominal pain during their presentation ( $A O R=4.28,95 \% \mathrm{Cl}=1.11$-15.52). The finding is in line with the study conducted in Kenya (25) and Turkey(45).

Similarly, time in which the patient get out of DKA was associated with longer hospital stay in this study. Those clients get out of DKA in greater than 72 hours were 6.39 times more likely to stay in hospital as compared with those clients get out of DKA less than 24 hours $(\mathrm{AOR}=6.39,95 \% \mathrm{Cl}=1.09-37.50)$. This might be due to differences in hyperglycemia state, clinical presentation and delay in management $(41,46)$.

In general, this study can bring out positive implications for clinical care, health service management and researches with in an area of diabetic specialization.

Clinically the health care worker can identify predictors associated with longer hospital stay among type one diabetic children admitted with DKA at clinical setup.

Health care managers can access current evidences about over all treatment outcome of DKA among diabetic children and to take remedial action to strengthen service delivery by the clinicians.

Researcher can also motivated to conduct further researches in this area by taking this study as preliminary findings.

\section{Conclusion}

In this study, majority (97.5\%) of admitted DKA clients were showed improvement and discharged to their home with 9.5 days mean length of hospital stay and with very low mortality rate but with high management complication $(14.2 \%)$

The study also suggests that, the time with in which the majority of the clients get out of DKA were found in between 24-48 hours.

Residence, family history of DM, glycaemia at admission, DKA precipitating factors, DKA presentation specifically, abdominal pain and time in which the patient get out of DKA were significantly associated with long hospital stay in the multivariable logistic regression model less than $5 \%$ level of significance.

Thus, to achieve the intended treatment out come early in time, modification of the approach and strategies by emphasizing the above predictors is mandatory with the recommendation of multicenter study in this regard.

\section{Limitation Of The Study}


Since the data were collected from medical records, detail treatment protocol and some of important laboratory results were not addressed. Another limitation is that, this study was conducted in one hospital only (Felege Hiwot comprehensive specialized referral hospital) which may be difficult to generalize the findings to another hospital in the region

\section{List Of Acronyms And Abbreviations}

ADA: American Diabetic Association; BGM: Blood Glucose Monitoring; BMI: Body Mass Index; CIRI: Continuous Intravenous Regular Insulin; DKA: Diabetic Keto Acidosis; ESPE: European Society for Pediatric Endocrinology; FBS: Fasting Blood Glucose; HIV: Human Immunodeficiency Virus; IDF: International Diabetic Federation; IHM: In Hospital Mortality; IVF: Intravenous Fluid; ISPAD: International Society of Pediatrics and Adolescent Diabetes; LHS: Length of Hospital Stay; NCDs: Non Communicable Diseases; RBS: Random Blood Glucose; SSA: Sub Saharan Africa; T1DM: Type one Diabetes Mellitus; TLC: Total Leucocyte Count; TB: Tuberculosis

\section{Declarations}

\section{Ethical approval and consent to participate}

Ethical clearance and approval were obtained from the institutional review board (IRB) of Bahir Dar University (IRB number 01-008).Written supportive letter was taken from pediatrics department of the hospitals on behalf of the patients. This study had no any danger or negative consequences for the study participants. Medical record numbers were used for the data collection and personal identifiers of the client were not used in this research report. Access to collected information was limited to the principal investigator and confidentiality had preserved throughout the time.

\section{Consent for publication}

Not applicable

\section{Availability of data and materials}

Data will be available upon consortium approval.

\section{Competing interests}

The author declared that, have no competing interests.

\section{Funding}

This research didn't receive grants from any funding agency in the public, commercial, or not-for-profit sectors

\section{Authors' contribution}

Fentahun Meseret had a substantial contribution from conception to the acquisition of the data. The author had a great contribution to the study design, analysis, and interpretation of the findings. The author drafted the manuscript and revised the drafted manuscript carefully for important intellectual contents and finally approved the final manuscript.

\section{Acknowledgment}

My earnest gratitude goes to institutional review board (IRB) of Bahir Dar University for their proper review and approval of this paper.

I would like to extend my acknowledgment to Felege Hiwot comprehensive specialized referral hospital staffs for their cooperation when i was in need of information related to this research writing.

My sincere gratitude and appreciation forward data collectors and participants without whom it would not be realized.

\section{Author information}

Haramaya University, college of health and medical science, school of nursing, and Midwifery, department of pediatrics and child health nursing, P.0. Box 235, Harar, Ethiopia.

\section{References}

1. OMS. Global Report on Diabetes. Isbn [Internet]. 2016;978:6-86. Available from:

http://www.who.int/about/licensing/copyright_form/index.html\%0Ahttp://www.who.int/about/licensing/copyright_form/index.html\%0Ahttps://apps.who.int/i

2. L. N, B. A, W. M, K. P. Determinants of outcome of children with type 1 diabetes in Cameroon. Horm Res Paediatr [Internet]. 2015;84:185. Available from: http://www.embase.com/search/results? subaction=viewrecord\&from=export\&id=L72085656\%0Ahttp://dx.doi.org/10.1159/000437032

3. Adamu Y, Garba KM, YAU S, Ya'u J. Glycemic Control and its Predictors among Diabetic Patients Attending a Tertiary Hospital in Nigeria: A Retrospective Observational Study. J Biomed Anal. 2020;3(1):13-25. 
4. Alhomood MA, Shibli KY, Abadi S. Knowledge about Diabetic Ketoacidosis among Parents of Type 1 Diabetic Children. World Fam Med Journal/Middle East J Fam Med. 2020;18(1):91-101.

5. Gomez-Lopera N, Pineda-Trujillo N, Diaz-Valencia PA. Correlating the global increase in type 1 diabetes incidence across age groups with national economic prosperity: A systematic review. World J Diabetes. 2019;10(12):560-80.

6. Onyiriuka AN, Ifebi E. Ketoacidosis at diagnosis of type 1 diabetes in children and adolescents: Frequency and clinical characteristics. J Diabetes Metab Disord. 2013;12(1).

7. Del Pozo P, Aránguiz D, Córdova G, Scheu C, Valle P, Cerda J, et al. Clinical profile of children with diabetic ketoacidosis in a critical care unit. Rev Chil Pediatr. 2018;89(4):491-8.

8. Mendez Y, Surani S, Varon J. Diabetic ketoacidosis: Treatment in the intensive care unit or general medical/surgical ward? World J Diabetes. $2017 ; 8(2): 40$.

9. J MAP, Varadarajan P. Risk factors for poor outcome in diabetic keto acidosis at the initial presentation among children with type 1 diabetes mellitus. 2018;5(5):1745-9.

10. Shenoy S, Upadhya N. the Factors Affecting Resolution of Acidosis in Children With Diabetic Ketoacidosis - a Retrospective Study From a Tertiary Care Center in India. Indian J Child Health. 2017;04(03):294-7.

11. Cashen K, Petersen T. Diabetic ketoacidosis. Pediatr Rev. 2019;40(8):412-20.

12. Phelan H, Lange K, Cengiz E, Gallego P, Majaliwa E, Pelicand J, et al. ISPAD Clinical Practice Consensus Guidelines 2018: Diabetes education in children and adolescents. Pediatr Diabetes. 2018;19(August):75-83.

13. Care P, Silberfein E, Perrier ND. M anagement of P heochromocytomas. 2009;579:579-84.

14. Ramírez-Mendoza F, González JE, Gasca E, Camacho M, Cruz M V., Caraveo D, et al. Time in range and HbA1C after 6 months with a multidisciplinary program for children and adolescents with diabetes mellitus, real world data from Mexico City. Pediatr Diabetes. 2020;21(1):61-8.

15. Kangin M, Talay MN, Tanriverdi Yilmaz S, Unal E, Demiral M, Asena M, et al. A Retrospective Analysis of Children and Adolescents With Diabetic Ketoacidosis in the Intensive Care Unit: Is It Significant that the Blood Ketone Level Becomes Negative in Diabetic Ketoacidosis? Cureus. 2020;12(10):1-8.

16. Shaltout AA, Channanath AM, Thanaraj TA, Omar D, Abdulrasoul M, Zanaty N, et al. Ketoacidosis at first presentation of type 1 diabetes mellitus among children: A study from Kuwait. Sci Rep [Internet]. 2016;6:1-9. Available from: http://dx.doi.org/10.1038/srep27519

17. Majaliwa ES, Mohn A, Chiavaroli V, Ramaiya K, Swai ABM, Chiarelli F. Management of diabetic ketoacidosis in children and adolescents in Sub-Saharan Africa: A review. East Afr Med J. 2010;87(4):167-73.

18. Atkilt HS, Turago MG, Tegegne BS. Clinical characteristics of diabetic ketoacidosis in children with newly diagnosed type 1 diabetes in Addis Ababa, Ethiopia: A cross-sectional study. PLoS One [Internet]. 2017;12(1):7-9. Available from: http://dx.doi.org/10.1371/journal.pone.0169666

19. Zeleke H, Murugan R, Wondwossen K. Incidence and predictors of diabetic ketoacidosis among children with diabetes in west and east gojjam zone referral hospitals north west Ethiopia ,. 2019;

20. McGregor S, Metzger DL, Amed S, Goldman RD. Fluid management in children with diabetic ketoacidosis. Can Fam Physician. 2020;66(11):817-9.

21. Raju G, Suma K. Outcome of children with diabetic ketoacidosis treated with a modified protocol. Asian Pacific J Heal Sci. 2017;4(4):160-2.

22. Ababulgu RZ, Tesfaye BT. Characteristics and Outcomes of Children with Type-I Diabetes Mellitus Hospitalized for Ketoacidosis. Curr Diabetes Rev. 2019;16(7):779-86.

23. Varadarajan P. Risk factors for mortality in children with diabetic keto acidosis from developing countries. World J Diabetes. 2014;5(6):932.

24. Broberg MCG, Rose JA, Slain KN. The Relationship Between Estimated Median Household Income and Critical Care Length of Stay in Children With Diabetic Ketoacidosis. Glob Pediatr Heal [Internet]. 2020;7. Available from: https://doi.org/10.1177/2333794X20956770

25. Musoma SN, Omar A, Mutai BC, Laigong P. Outcomes of Children and Adolescents Admitted with Diabetic Ketoacidosis at Kenyatta National Hospital (KNH), Kenya. J Diabetes Res. 2020;2020:8987403.

26. J. MAP, Varadarajan P. Risk factors for poor outcome in diabetic keto acidosis at the initial presentation among children with type 1 diabetes mellitus. Int J Contemp Pediatr. 2018;5(5):1745.

27. De Vries L, Oren L, Lazar L, Lebenthal Y, Shalitin S, Phillip M. Factors associated with diabetic ketoacidosis at onset of Type 1 diabetes in children and adolescents. Diabet Med. 2013;30(11):1360-6. 
28. Castellanos L, Tuffaha M, Koren D, Levitsky LL. Management of Diabetic Ketoacidosis in Children and Adolescents with Type 1 Diabetes Mellitus. Pediatr Drugs [Internet]. 2020;22(4):357-67. Available from: https://doi.org/10.1007/s40272-020-00397-0

29. Choi HS, Kwon A, Chae HW, Suh J, Kim DH, Kim HS. Respiratory failure in a diabetic ketoacidosis patient with severe hypophosphatemia. Ann Pediatr Endocrinol Metab. 2018;23(2):103-6.

30. Moraes AG de, Surani S. Effects of diabetic ketoacidosis in the respiratory system. World J Diabetes. 2019;10(1):16-22.

31. Yaneva NY, Konstantinova MM, lliev DI. Risk factors for cerebral oedema in children and adolescents with diabetic ketoacidosis. Biotechnol Biotechnol Equip [Internet]. 2016;30(6):1142-7. Available from: http://dx.doi.org/10.1080/13102818.2016.1221740

32. Murunga AN, Owira PMO. Diabetic ketoacidosis: An overlooked child killer in sub-Saharan Africa? Trop Med Int Heal. 2013;18(11):1357-64.

33. Huang SK, Huang CY, Lin CH, Cheng BW, Chiang YT, Lee YC, et al. Acute kidney injury is a common complication in children and adolescents hospitalized for diabetic ketoacidosis. PLoS One [Internet]. 2020;15(10 October):1-10. Available from: http://dx.doi.org/10.1371/journal.pone.0239160

34. Frequency, clinical characteristics, biochemical findings and outcomes of DKA at the onset of type-1 DM in young children and adolescents living in a developing country - an experience from a pediatric emergency department. 2019;32(2):2019.

35. Tieder JS, McLeod L, Keren R, Luan X, Localio R, Mahant S, et al. Variation in resource use and readmission for diabetic ketoacidosis in children's hospitals. Pediatrics. 2013;132(2):229-36.

36. Islam R, Akhter S, Shelim R, Mohsin F, Begum T, Akhter G. Precipitating factors, clinical features and outcome of diabetic ketoacidosis in children and adolescents admitted in a tertiary care hospital in Dhaka. Bangladesh J Med Sci. 2014;13(1):53-7.

37. Ali SH, Mohammed MM, Youssef RS. The Pattern of Electrolyte Imbalance in Critically III Children admitted in Pediatric Emergency Unit at Sohag University Hospital. 2021;82(January):276-81.

38. El R, Mohammed S, Monemabdalla N El. Clinical , and Biochemical Profile of Pediatric Diabetic Ketoacidosis Patients in Al-zahraa Hospital Pediatric ICU. 2018;73(October):7507-11.

39. Ahmed AM, Khabour OF, Ahmed SM, Alebaid IA, Ibrahim AM. Frequency and severity of ketoacidosis at diagnosis among childhood type 1 diabetes in khartoum state, sudan. Afr Health Sci. 2020;20(2):841-8.

40. Hadgu FB, Sibhat GG, Gebretsadik LG. <p>Diabetic ketoacidosis in children and adolescents with newly diagnosed type 1 diabetes in Tigray, Ethiopia: retrospective observational study</p>. Pediatr Heal Med Ther. 2019;Volume 10:49-55.

41. Lopes CLS, Pinheiro PP, Barberena LS, Eckert GU. Cetoacidose diabética em uma unidade de terapia intensiva pediátrica. J Pediatr (Rio J) [Internet]. 2017;93(2):179-84. Available from: http://dx.doi.org/10.1016/j.jped.2016.05.008

42. Matthews JA. Medical Science. Encycl Environ Chang. 2014;22(October 2017).

43. Cameron FJ, Scratch SE, Nadebaum C, Northam EA, Koves I, Jennings J, et al. Neurological Consequences of Diabetic Ketoacidosis at Initial Presentation of Type 1 Diabetes in a Prospective Cohort Study of Children. 2014;37(June):1554-62.

44. Razavi Z, Hamidi F. Diabetic ketoacidosis: Demographic data, clinical profile and outcome in a tertiary care hospital. Iran J Pediatr. 2017;27(3):0-5.

45. Turan C, Yurtseven A, Basa EG, Gökşen D, Saz EU. The effects of prehospital care on outcome in pediatric diabetic ketoacidosis. JCRPE J Clin Res Pediatr Endocrinol. 2020;12(2):189-96.

46. Varshney GA, Varshney D, Mehr V, Kela G, Kharia R, Agrawal G, et al. Clinical Profile and Outcome of Diabetic Ketoacidosis in Children At Tertiary Care Hospital. J Evol Med Dent Sci. 2015;4(31):5329-33.

47. Ghetti S, Kuppermann N, Rewers A, Myers SR, Schunk JE, Stoner MJ, et al. Cognitive function following diabetic ketoacidosis in children with new-onset or previously diagnosed type 1 diabetes. Diabetes Care. 2020;43(11):2768-75.

48. Ferreira JP, Ferraro M. Clinical trial of fluid infusion rates for pediatric diabetic ketoacidosis. Arch Argent Pediatr. 2018;116(6):E793-4.

49. Hasan RA, Hamid K, Dubre D, nolan brian, Sharman M. The Two-bag System for Intravenous Fluid Management of Children with Diabetic Ketoacidosis: Experience from a Community-Based Hospital. Glob Pediatr Heal [Internet]. 2021;8. Available from: https://doi.org/10.1177/2333794X21991532

50. Della Manna T, Schvartsman C, Steinmetz L, Kuperman H, Campos PR, Setian N, et al. Subcutaneous Use of a Fast-Acting. Diabetes Care. 2005;28(8):1856-61.

51. Uleanya ND, Aniwada EC, Nwokoye IC, Ndu IK, Eke CB. Relationship between Glycemic Levels and Treatment Outcome among Critically III Children admitted into Emergency Room in Enugu. BMC Pediatr. 2017;17(1):1-7. 
52. Alemu Desse T, Eshetie T. Determinants of Long Hospital Stay among Diabetic Patients Admitted with Diabetic Ketoacidosis at Jimma University Specialized Hospital. J Trauma Stress Disord Treat. 2017;06(01):1-4.

\section{Tables}

Table 1: sociodemographic characteristics among type 1 diabetic children admitted with DKA, Bahir Dar, Ethiopia, 2021(n=162)

\begin{tabular}{llll} 
Variable & Category & Number(N) & Percent (\%) \\
\hline Age group in years & $<=5$ & 33 & 20.4 \\
\cline { 2 - 3 } & $>5-10$ & 50 & 30.9 \\
\cline { 2 - 3 } Sex & $>10-14$ & 79 & 48.8 \\
\hline \multirow{2}{*}{ Resident } & Male & 83 & 51.2 \\
\cline { 2 - 3 } & Female & 79 & 48.8 \\
\hline Educational status of children & Urban & 54 & 33.3 \\
\cline { 2 - 3 } & Rural & 108 & 66.7 \\
\cline { 2 - 4 } & Primary school & 96 & 30.3 \\
\hline Primary care giver & High school & 17 & 59.3 \\
& Mother & 80 & 10.5 \\
& Father & 27 & 49.4 \\
& His/her self & 25 & 16.7 \\
& Other & 30 & 15.4 \\
& & & 18.5
\end{tabular}

Table 2: Clinical characteristics among type 1 diabetic children admitted with DKA, Bahir Dar, 2021(n=162) 


\begin{tabular}{|c|c|c|c|}
\hline Variable & Category & Number(N) & Percent (\%) \\
\hline \multirow[t]{2}{*}{ Diagnosis } & Newly & 130 & 80.2 \\
\hline & Known & 32 & 19.8 \\
\hline \multirow[t]{3}{*}{ BMI } & $<16 \mathrm{~kg} / \mathrm{m} 2$ & 89 & 54.9 \\
\hline & $16-25 \mathrm{~kg} / \mathrm{m} 2$ & 71 & 43.8 \\
\hline & $>25 \mathrm{~kg} / \mathrm{m} 2$ & 2 & 1.2 \\
\hline \multirow[t]{2}{*}{ Family history of DM } & NO & 137 & 84.6 \\
\hline & Yes & 25 & 15.4 \\
\hline \multirow[t]{2}{*}{ Duration of DM(years) } & $<=3$ & 76 & 46.9 \\
\hline & $>3$ & 86 & 53.1 \\
\hline \multirow[t]{3}{*}{ Insulin regimen } & Regular\&lent & 103 & 63.6 \\
\hline & Regular\&NPH & 30 & 18.5 \\
\hline & NPH alone & 29 & 17.9 \\
\hline \multirow[t]{2}{*}{ Insulin dose } & $<=7 \mathrm{u} / \mathrm{kg} / \mathrm{d}$ & 75 & 46.3 \\
\hline & $>7 \mathrm{u} / \mathrm{kg} / \mathrm{d}$ & 87 & 53.7 \\
\hline \multirow[t]{2}{*}{ Frequency of glycemic control } & $<3$ & 50 & 30.9 \\
\hline & $>=3$ & 112 & 69.1 \\
\hline \multirow[t]{2}{*}{ History of comorbidity } & NO & 54 & 33.3 \\
\hline & Yes & 108 & 66.7 \\
\hline \multirow[t]{2}{*}{ Autoimmune disease } & NO & 159 & 98.1 \\
\hline & Yes & 3 & 1.9 \\
\hline \multirow[t]{2}{*}{ Hypertension(HTN) } & NO & 160 & 98.8 \\
\hline & Yes & 2 & 1.2 \\
\hline \multirow[t]{2}{*}{ Cardio vascular disease(CVD) } & NO & 161 & 99.4 \\
\hline & Yes & 1 & 0.6 \\
\hline Wasting & NO & 113 & 69.8 \\
\hline \multirow[t]{2}{*}{ Stunting } & Yes & 49 & 30.2 \\
\hline & NO & 149 & 92.0 \\
\hline \multirow[t]{2}{*}{ Pneumonia } & Yes & 13 & 8.0 \\
\hline & NO & 136 & 84.0 \\
\hline \multirow[t]{2}{*}{ Urinary tract infection(UTI) } & Yes & 26 & 16.0 \\
\hline & NO & 138 & 85.2 \\
\hline \multirow[t]{2}{*}{ Upper respiratory tract infection(URTI) } & Yes & 24 & 14.8 \\
\hline & NO & 152 & 93.8 \\
\hline \multirow[t]{2}{*}{ Tuberculosis(TB) } & Yes & 10 & 6.2 \\
\hline & NO & 159 & 98.2 \\
\hline \multirow[t]{2}{*}{ Acute gastroenteritis(AGE) } & Yes & 3 & 1.8 \\
\hline & NO & 145 & 89.5 \\
\hline Meningitis & Yes & 17 & 10.5 \\
\hline \multirow[t]{2}{*}{ Hepatitis } & NO & 157 & 96.9 \\
\hline & Yes & 5 & 3.1 \\
\hline \multicolumn{4}{|l|}{ Malaria } \\
\hline & NO & 158 & 97.5 \\
\hline
\end{tabular}




$\begin{array}{llll}\text { Anemia } & \text { Yes } & 4 & 2.5 \\ & & & \\ & \text { NO } & 155 & 95.7 \\ \text { Fungal infection } & \text { Yes } & 7 & 4.3 \\ & & & \\ \text { Other comorbidity } & \text { NO } & 152 & 93.8 \\ & \text { Yes } & 10 & 6.2 \\ & \text { NO } & 145 & 89.5 \\ \text { More than one comorbid illness } & \text { Yes } & 17 & 10.5 \\ & \text { NO } & 154 & 95.1 \\ & \text { Yes } & 8 & 4.9 \\ & \text { NO } & 97 & 59.9 \\ & \text { Yes } & 65 & 40.1\end{array}$

Table 3: Frequency of DKA episode, severity and its precipitating factors among type 1 diabetic children admitted with DKA, Bahir Dar, 2021( $n=162)$

\begin{tabular}{|c|c|c|c|}
\hline Variable & Category & Number(N) & Percent (\%) \\
\hline \multirow[t]{3}{*}{ Frequency of DKA episode } & Once & 116 & 71.6 \\
\hline & Two times & 31 & 19.1 \\
\hline & Three times & 12 & 7.4 \\
\hline \multirow[t]{3}{*}{ Severity of DKA } & Four times & 3 & 1.9 \\
\hline & Mild & 72 & 44.4 \\
\hline & Moderate & 61 & 37.7 \\
\hline \multirow[t]{4}{*}{ Precipitating factors } & Sever & 29 & 17.9 \\
\hline & Infection & 91 & 56.2 \\
\hline & Insulin skipping/omission & 13 & 8.1 \\
\hline & Insulin defaulter & 23 & 14.2 \\
\hline \multirow[t]{5}{*}{ Location of admission } & In appropriate insulin storage & 15 & 9.3 \\
\hline & Unspecified/not documented & 38 & 23.5 \\
\hline & Emergency only & 24 & 14.8 \\
\hline & Intensive care unit(ICU) & 11 & 6.8 \\
\hline & Pediatrics ward & 127 & 78.4 \\
\hline
\end{tabular}

NB: The most common infection that precipitates DKA includes urinary tract infection, upper respiratory tract infection, acute gastro enteritis, pneumonia etc. See above in Table 2.

Table 4: Clinical presentation and Laboratory results among type 1 diabetic children admitted with DKA, Bahir Dar, 2021(n=162) 


\begin{tabular}{|c|c|c|c|}
\hline Variable & Category & Number(N) & Percent (\%) \\
\hline \multirow[t]{2}{*}{ Polyuria } & NO & 18 & 11.1 \\
\hline & Yes & 144 & 88.9 \\
\hline \multirow[t]{2}{*}{ Polydipsia } & NO & 25 & 15.4 \\
\hline & Yes & 137 & 84.6 \\
\hline \multirow[t]{2}{*}{ Polyphagia } & NO & 101 & 62.4 \\
\hline & Yes & 61 & 37.6 \\
\hline \multirow[t]{2}{*}{ Abdominal pain } & NO & 111 & 68.5 \\
\hline & Yes & 51 & 31.5 \\
\hline \multirow[t]{2}{*}{ Nausea/vomiting } & NO & 99 & 61.1 \\
\hline & Yes & 63 & 38.9 \\
\hline \multirow[t]{2}{*}{ Fatigue } & NO & 99 & 61.1 \\
\hline & Yes & 63 & 38.9 \\
\hline \multirow[t]{2}{*}{ Cough } & NO & 239 & 85.8 \\
\hline & Yes & 23 & 14.2 \\
\hline \multirow[t]{2}{*}{ Weight loss } & NO & 115 & 71 \\
\hline & Yes & 47 & 29 \\
\hline \multirow[t]{2}{*}{ Respiratory distress } & NO & 125 & 77.2 \\
\hline & Yes & 37 & 22.8 \\
\hline \multirow[t]{2}{*}{ Confusion } & NO & 137 & 84.6 \\
\hline & Yes & 25 & 15.4 \\
\hline \multirow[t]{2}{*}{ Blurred vision } & NO & 156 & 96.3 \\
\hline & Yes & 6 & 3.7 \\
\hline \multirow[t]{2}{*}{ Fever } & NO & 154 & 95.1 \\
\hline & Yes & 8 & 4.9 \\
\hline \multirow[t]{2}{*}{ Others } & NO & 156 & 96.3 \\
\hline & Yes & 6 & 3.7 \\
\hline PR(beats/min) & & $115 \pm 22.1$ & \\
\hline RR(breath/min) & & $31.1 \pm 10.3$ & \\
\hline Temperature $\left({ }^{0} \mathrm{c}\right)$ & & $36.6 \pm 0.9$ & \\
\hline Duration of chief complaint in days & & $14.1 \pm 16.1$ & \\
\hline Glycaemia at admission(mg/dl) & & $508.7 \pm 101.8$ & \\
\hline \multicolumn{4}{|l|}{ Ketone level(mmol/l) } \\
\hline \multirow[t]{2}{*}{ Urine glucose level(mmol/l) } & & $2.7 \pm 0.9$ & \\
\hline & & $2.4 \pm 0.8$ & \\
\hline
\end{tabular}

PR-Pulse Rate, RR-Respiration Rate; Others include diarrhea, headache and dizziness

Table 5: Management protocol and its treatment outcome among type 1 diabetic children admitted with DKA, Bahir Dar, 2021(n=162) 


\begin{tabular}{|c|c|c|c|}
\hline Variable & Category & Number(N) & Percent (\%) \\
\hline Types of IV fluid bolus & Normal saline(NS) & 161 & 99.4 \\
\hline Amount of IV fluid bolus in milliliter(Mean \pm SD) & Ringer lactate(RL) & 1 & 0.6 \\
\hline \multirow[t]{5}{*}{ Types of MF used in the management } & & $362.2 \pm 348.4$ & \\
\hline & NS only & 63 & 38.9 \\
\hline & NS\&D5W & 75 & 46.3 \\
\hline & NS\&D10\% & 6 & 3.7 \\
\hline & NS,D5W\&D10\% & 2 & 1.2 \\
\hline Rate of MF used during management in $\mathrm{ml} / \mathrm{hr}$.(Mean \pm SD) & NS,D5W\&D40\% & 2 & 1.2 \\
\hline \multicolumn{4}{|l|}{ Insulin administration rate (u/hr.) } \\
\hline \multicolumn{2}{|l|}{ Regular insulin } & \multicolumn{2}{|l|}{$89 \pm 14$} \\
\hline \multicolumn{4}{|l|}{ Potassium replacement } \\
\hline & & \multicolumn{2}{|l|}{$9.6 \pm 4.3 \mathrm{u}$} \\
\hline \multirow[t]{3}{*}{ Time in which the patient get out of DKA in hours } & & 162 & 100 \\
\hline & NO & 106 & 65.4 \\
\hline & Yes & 56 & 34.6 \\
\hline \multirow[t]{8}{*}{ Complication } & $<24$ & 33 & 20.4 \\
\hline & $24-48$ & 58 & 35.8 \\
\hline & $48-72$ & 38 & 23.5 \\
\hline & $>72$ & 33 & 20.4 \\
\hline & Hypokalemia & 11 & 6.8 \\
\hline & Hyperkalemia & 2 & 1.2 \\
\hline & Hypernatremia & 4 & 2.5 \\
\hline & Hyponatremia & 5 & 3.1 \\
\hline Length of hospital stay & Neurologic sequali & 4 & 2.5 \\
\hline Average length of hospital stay & Hyperchloremia & 3 & 1.9 \\
\hline Minimum length of hospital stay & Hypochloremia & 1 & 0.6 \\
\hline Maximum length of hospital stay & AKI & 3 & 1.9 \\
\hline \multirow[t]{7}{*}{ Overall treatment outcome } & $<=7$ & 66 & 40.7 \\
\hline & $>7$ & 96 & 59.3 \\
\hline & & \multicolumn{2}{|l|}{$9.5 \pm 6.2$ days } \\
\hline & & \multicolumn{2}{|l|}{1 day } \\
\hline & & \multicolumn{2}{|l|}{35 days } \\
\hline & Not improved/died & 4 & 2.5 \\
\hline & Improved & 158 & 97.5 \\
\hline
\end{tabular}




\begin{tabular}{|c|c|c|c|}
\hline Variables & $\operatorname{coR}(95 \% \mathrm{Cl})$ & $\operatorname{AOR}(95 \% \mathrm{Cl})$ & $\mathrm{p}$-value \\
\hline \multicolumn{4}{|l|}{ Age group } \\
\hline \multicolumn{4}{|l|}{$<=5 \circledast$} \\
\hline$>5-10$ & $1.66(0.68-4.02)$ & 2.93(0.63-13.66) & 0.171 \\
\hline$>10-14$ & $2.31(1.01-5.29)$ & $5.51(0.92-33.02)$ & 0.062 \\
\hline \multicolumn{4}{|l|}{ Residence } \\
\hline Rural & $3.17(1.61-6.24)$ & $4.31(1.25-14.80)$ & $0.020 *$ \\
\hline \multicolumn{4}{|l|}{ Urban $®$} \\
\hline \multicolumn{4}{|l|}{ Educational status of children } \\
\hline \multicolumn{4}{|l|}{$\mathrm{KG} /$ not started $\circledast$} \\
\hline primary school & $1.67(0.83-3.36)$ & $0.21(0.02-2.01)$ & 0.175 \\
\hline secondary school & $1.37(0.45-4.19)$ & $0.58(0.02-11.40)$ & 0.663 \\
\hline Body mass index & $0.88(0.79-0.97)$ & $0.96(0.79-1.17)$ & 0.682 \\
\hline Duration of chief complaint & $1.01(0.99-1.04)$ & $0.99(0.96-1.03)$ & 0.901 \\
\hline \multicolumn{4}{|l|}{ Diagnosis } \\
\hline known diabetes & $0.46(0.21-0.99)$ & $0.51(0.13-2.02)$ & 0.334 \\
\hline \multicolumn{4}{|l|}{ Newly diagnosis ${ }^{\circledR}$} \\
\hline \multicolumn{4}{|l|}{ Family history of diabetes } \\
\hline Yes & $0.48(0.20-1.14)$ & $0.12(0.02-0.64)$ & $0.013^{*}$ \\
\hline \multicolumn{4}{|l|}{$\mathrm{NO} \circledast$} \\
\hline Glycaemia at admission & $1.01(1.00-1.02)$ & $1.01(1.00-1.02)$ & $0.008^{*}$ \\
\hline \multicolumn{4}{|l|}{ Severity of DKA } \\
\hline \multicolumn{4}{|l|}{ Mild $\AA$} \\
\hline Moderate & $1.70(0.84-3.44)$ & $0.72(0.21-2.44)$ & 0.599 \\
\hline Sever & $1.46(0.61-3.53)$ & $0.41(0.08-2.06)$ & 0.281 \\
\hline \multicolumn{4}{|l|}{ Frequency of DKA episode } \\
\hline \multicolumn{4}{|l|}{ One® } \\
\hline Two & $1.43(0.63-3.25)$ & $0.61(0.14-2.64)$ & 0.506 \\
\hline Three & 3.92(0.82 -18.70 & $3.28(0.42-25.43)$ & 0.255 \\
\hline Four & $0.39(0.03-4.45)$ & $0.02(0.01-1.96)$ & 0.094 \\
\hline \multicolumn{4}{|l|}{ DKA Precipitating factors } \\
\hline \multicolumn{4}{|l|}{ Infection ${ }^{\circledR}$} \\
\hline Insulin skipping/omission & $0.18(0.05-0.68)$ & $0.08(0.01-0.98)$ & $0.048^{*}$ \\
\hline Insulin defaulter & $0.38(0.16-0.95)$ & $1.19(0.14-10.06)$ & 0.871 \\
\hline Not documented & $0.68(0.33-1.37)$ & $1.46(0.35-6.05)$ & 0.603 \\
\hline \multicolumn{4}{|c|}{ DKA presentation during admission } \\
\hline \multicolumn{4}{|l|}{ Polydipsia } \\
\hline Yes & $1.72(0.73-4.04)$ & $0.79(0.17-3.75)$ & 0.766 \\
\hline \multicolumn{4}{|l|}{$\mathrm{NO} \circledast$} \\
\hline \multicolumn{4}{|l|}{ Abdominal pain } \\
\hline Yes & $1.58(0.79-3.16)$ & $4.28(1.11-15.52)$ & $0.035^{\star}$ \\
\hline \multicolumn{4}{|l|}{$\mathrm{NO} \circledast$} \\
\hline \multicolumn{4}{|l|}{ Confusion } \\
\hline Yes & $1.95(0.76-4.96)$ & $2.01(0.41-9.89)$ & 0.389 \\
\hline
\end{tabular}

Page 16/19 


\begin{tabular}{|c|c|c|c|}
\hline \multicolumn{4}{|l|}{$\mathrm{NO} \circledast$} \\
\hline Body temperature & $0.68(0.46-1.01)$ & $0.84(0.43-1.65)$ & 0.612 \\
\hline \multicolumn{4}{|l|}{ Comorbid illness } \\
\hline Yes & $1.62(0.85-3.11)$ & $0.63(0.12-3.26)$ & 0.579 \\
\hline \multicolumn{4}{|l|}{$\mathrm{NO} \circledast$} \\
\hline \multicolumn{4}{|c|}{ Urinary tract infection } \\
\hline Yes & $3.01(1.06-8.52)$ & $0.98(0.17-5.76)$ & 0.985 \\
\hline \multicolumn{4}{|l|}{$\mathrm{NO} \circledast$} \\
\hline \multicolumn{4}{|c|}{ Upper respiratory tract infection } \\
\hline Yes & $0.39(0.09-1.71)$ & $1.57(0.09-26.47)$ & 0.755 \\
\hline \multicolumn{4}{|l|}{$\mathrm{NO} \circledast$} \\
\hline \multicolumn{4}{|l|}{ Wasting } \\
\hline Yes & $3.28(1.52-7.05)$ & $1.68(0.38-6.78)$ & 0.526 \\
\hline \multicolumn{4}{|l|}{$\mathrm{NO} \circledast$} \\
\hline \multicolumn{4}{|l|}{ Stunting } \\
\hline Yes & $2.44(0.66-9.24)$ & $1.29(0.12-13.75)$ & 0.831 \\
\hline \multicolumn{4}{|l|}{$\mathrm{NO} \circledast$} \\
\hline \multicolumn{4}{|l|}{ Fungal infection } \\
\hline Yes & $2.43(0.76-7.81)$ & $2.63(0.28-25.12)$ & 0.401 \\
\hline \multicolumn{4}{|l|}{$\mathrm{NO} \circledast$} \\
\hline \multicolumn{4}{|l|}{ Comedication } \\
\hline Yes & $1.97(1.01-3.84)$ & $2.08(0.54-7.98)$ & 0.284 \\
\hline \multicolumn{4}{|l|}{$\mathrm{NO} \circledast$} \\
\hline \multicolumn{4}{|c|}{ Location of admission } \\
\hline \multicolumn{4}{|l|}{ Emergency ${ }^{\circledR}$} \\
\hline ICU & $1.14(0.26-5.09)$ & $0.29(0.02-3.65)$ & 0.337 \\
\hline Ward & $3.91(1.55-9.85)$ & $4.78(1.09-20.97)$ & 0.05 \\
\hline \multicolumn{4}{|c|}{ Time in $w / h$ the patient get out of DKA } \\
\hline \multicolumn{4}{|l|}{$<24 \mathrm{hr} \circledast$} \\
\hline $24-48 \mathrm{hr}$ & $2.86(1.18-6.94)$ & $0.86(0.28-3.79)$ & 0.847 \\
\hline $48-72 \mathrm{hr}$ & $2.68(1.02-7.02)$ & $1.04(0.22-4.84)$ & 0.964 \\
\hline$>72 \mathrm{hr}$ & $5.47(1.88-15.88)$ & $6.39(1.09-37.50)$ & $0.040 *$ \\
\hline \multicolumn{4}{|c|}{ Management Complication } \\
\hline Yes & $2.15(0.80-5.79)$ & $1.92(0.37-9.88)$ & 0.437 \\
\hline $\mathrm{NO} \circledast$ & & & \\
\hline
\end{tabular}

Where, ${ }^{\circledR}=$ Reference group; ${ }^{*}=$ significantly associated variables at a point less than 0.05

\section{Figures}




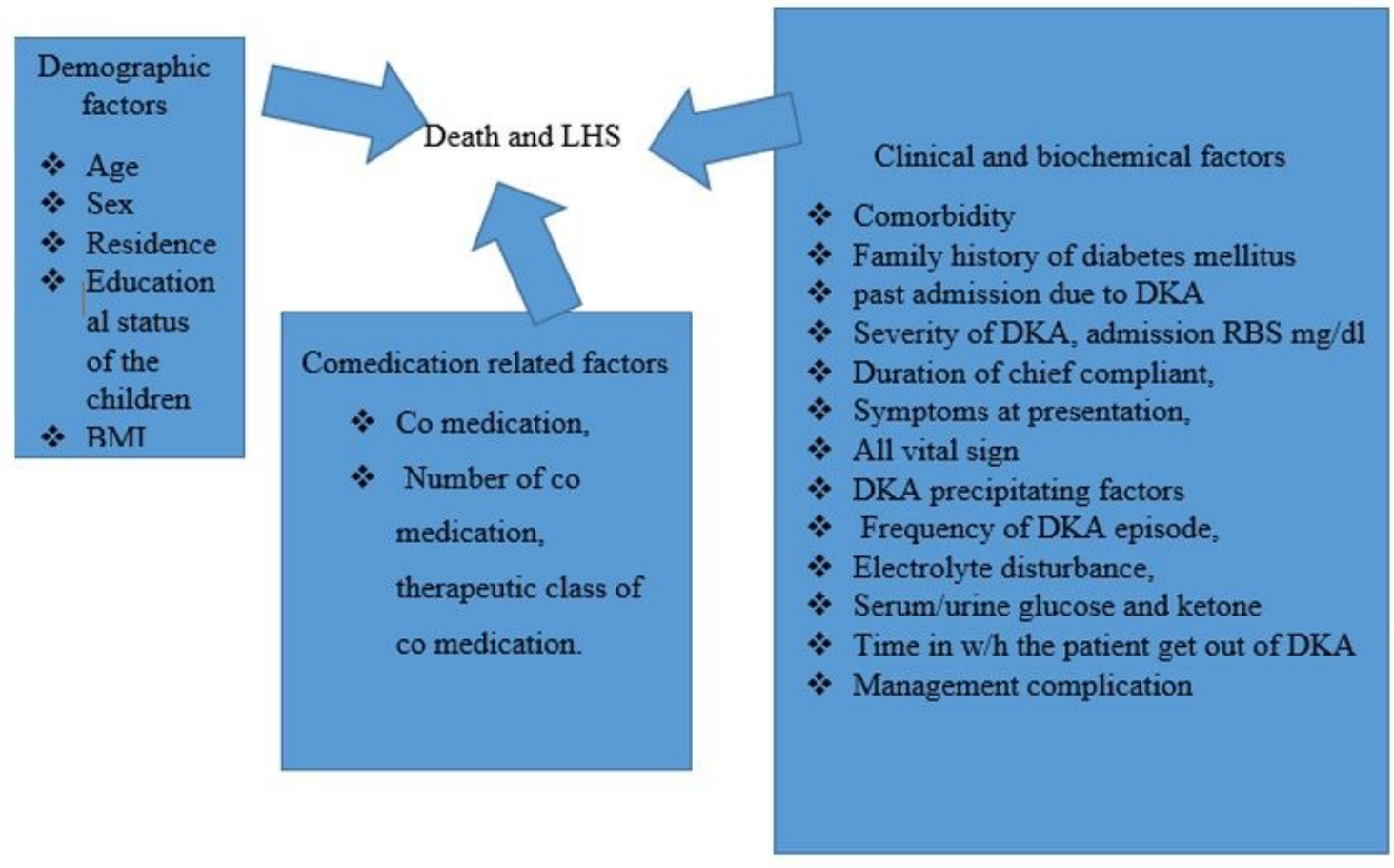

Figure 1

Factors affecting DKA treatment outcome concept map among type 1 diabetic children in Bahir Dar city Felege Hiwot referral hospitals, Northwest, Ethiopia, 2021; adapted from(22),(23), (25), (36, (44)).

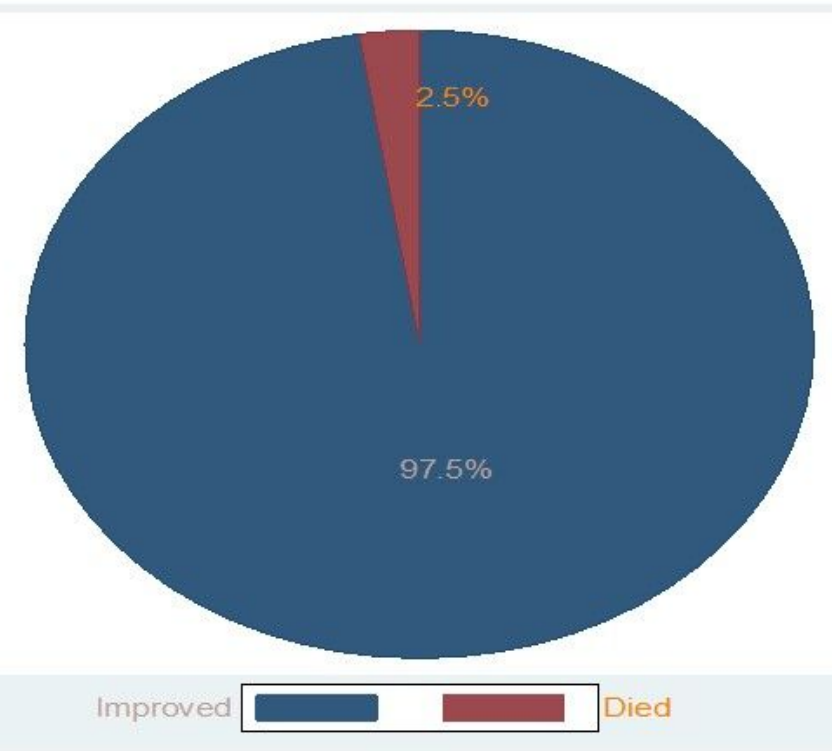

\section{Figure 2}

Over all treatment outcome among type 1 diabetic children admitted with DKA, Bahir Dar, 2021( $n=162)$ 


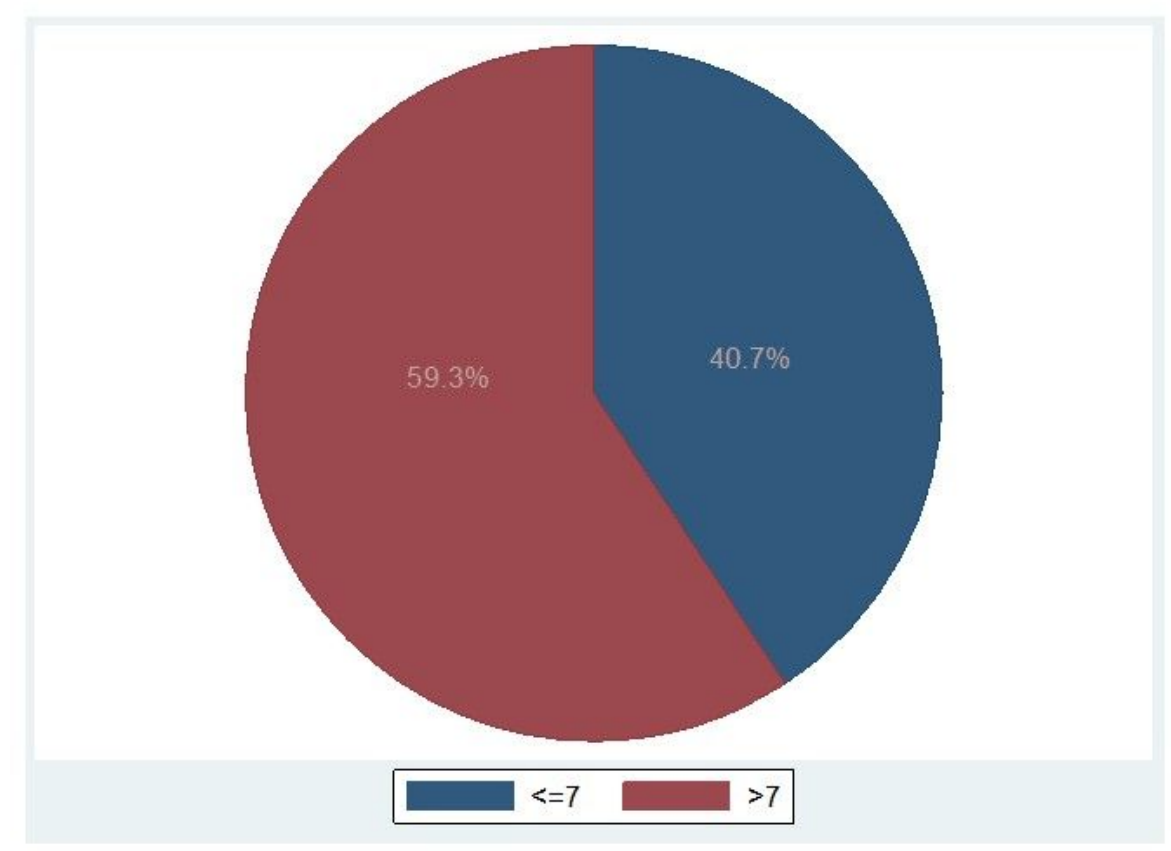

\section{Figure 3}

Proportion of clients stayed in the hospital for greater than seven days among type 1 diabetic children admitted with DKA, Bahir Dar, 2021( $\mathrm{n}=162$ )

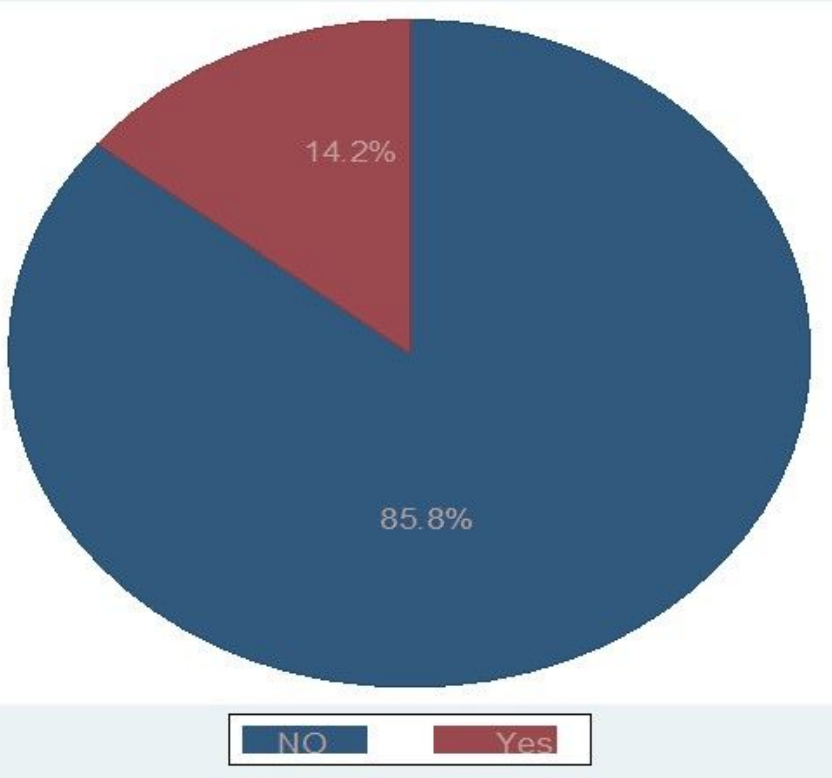

\section{Figure 4}

Proportion of clients who developed complication management among type 1 diabetic children admitted with DKA, Bahir Dar, 2021(n=162) 